\title{
À qui incombe le patrimoine religieux québécois ?
}

Who's responsible for Quebec's religious heritage?

\section{Violette Loget et Yves Bergeron}

\section{CpenEdition}

\section{Journals}

Édition électronique

URL : http://journals.openedition.org/iss/1713

DOI : $10.4000 /$ iss. 1713

ISSN : 2306-4161

\section{Éditeur}

ICOM - International Council of Museums

\section{Édition imprimée}

Date de publication : 12 octobre 2019

Pagination : 139-155

ISBN : 978-92-9012-467-2

ISSN : 2309-1290

\section{Référence électronique}

Violette Loget et Yves Bergeron, «À qui incombe le patrimoine religieux québécois ? », ICOFOM Study Series [En ligne], 47(1-2) | 2019, mis en ligne le 12 octobre 2019, consulté le 24 octobre 2019. URL http://journals.openedition.org/iss/1713 ; DOI : 10.4000/iss.1713 


\section{À qui incombe le patrimoine religieux québécois?}

\section{Violette Loget}

avec la collaboration de Yves Bergeron Université du Québec à Montréal - Montréal, Canada et Université Sorbonne Nouvelle Paris 3 Paris, France

Les catholiques qui sont nés vers 1950 ont prié et chanté en latin dans des églises aux odeurs d'encens derrière un prêtre qui officiait une liturgie venue tout droit du concile de Trente. Vingt ans aprés, la messe se disait en français pendant que l'orfévrerie et les vêtements brodés, jugés trop ostentatoires par le renouveau issu du concile Vatican II, étaient relégués aux oubliettes.

Jean Simard (Turgeon, 2005, p. 547)

RÉSUMÉ

Cette étude rend compte des rapports ambigus, oscillant de la coopération à la concurrence, entre l'Église, l'État et les musées québécois au regard de l'administration et de la protection du patrimoine matériel religieux, spécifiquement en contexte d'aliénation.

Mots clés : Patrimoine religieux, Québec, Protection, Politique, Aliénation 
Who's responsible for Quebec's religious heritage?

This study focuses on the ambiguous relationship, alternating between cooperation and competition, between Quebec's Church, State and museums in relation to the administration and the protection of tan-

gible religious heritage, particularly in the context of disposal.

Key words: Religious Heritage, Quebec, Protection, Politics, Disposal

丈

\section{La situation du patrimoine religieux québécois}

Depuis la fin du $\mathrm{XX}^{\mathrm{e}}$ siècle, le patrimoine religieux, qui avait historiquement rayonné dans les sociétés occidentales chrétiennes, est considéré en crise. Les spécialistes mettent en garde contre les risques de disparition, de dispersion et de dissociation des biens du clergé (Turgeon, 2005; Paine, 2014 ; Minucciani, 20I3; Roque, 20II). Au Québec, cette situation touche particulièrement l'Église catholique, dont

[l]e patrimoine religieux est le patrimoine le plus ancien et le plus riche du Québec, plus particulièrement pour les francophones catholiques qui constituent $85 \%$ de la population (Turgeon, 2005, p. 18) ${ }^{1}$.

Depuis les années I990, on recense de multiples initiatives pour protéger ce patrimoine en péril. Au-delà d'actions citoyennes, pensons aux travaux de Jean-Claude Marsan de l'École d'architecture de l'Université de Montréal sur les stratégies de réaménagement d'églises, de Luc Noppen et Lucie K. Morisset de l'Université du Québec à Montréal sur le patrimoine bâti, ainsi que de Laurier Turgeon et Jean Simard de l'Université Laval sur le patrimoine religieux matériel et immatériel. D'importants colloques jalonnent les trente dernières années avec «L'avenir des biens d'Église » en I997, «Entre le cultuel et le culturel » en 2004 ou encore « Avant de bazarder le patrimoine religieux! » en 20I8. Ce patrimoine fait l'objet d'une attention particulière dans les rapports publics : en 2000, Notre patrimoine, un présent du passé soulignait l'importance

I. Cette préoccupation est davantage présente au sein de la communauté canadienne francophone en raison de ses racines catholiques, alors que les Canadiens anglophones ont une culture protestante. 
de la conservation, de l'étude et de la diffusion des patrimoines religieux du Québec (Arpin, 200o, p. ro), et douze ans plus tard les Recommandations adoptées lors du Grand Chantier des États généraux des musées du Québec comptaient le patrimoine religieux au nombre des patrimoines à sauvegarder, «déjà en voie de disparition » et devenant «peu à peu "orphelin" des communautés qui le détiennent » (20I2, p. 48).

À l'occasion du $4 \mathrm{I}^{\text {ème }}$ symposium d'ICOFOM consacré à « la muséologie et [au] sacré » réfléchissons à deux enjeux interconnectés : la protection et la politique encadrant la gestion des biens catholiques québécois. Et ce, à travers l'étude de stratégies d'aliénation afin de cerner certaines des règles écrites et tacites des acteurs responsables de ce patrimoine matériel. Dans un premier temps, nous proposons un survol de l'état de l'administration dudit patrimoine dans le système québécois qui répond d'une philosophie de « laïcité-collaboration » selon Ernest Caparros ${ }^{1}$, en rendant compte des initiatives - isolées ou complémentaires - de ses gardiens (Église, État et musées nationaux) et en présentant succinctement l'état du droit civil et canonique s'appliquant en la matière. Nous exposerons ensuite la complexité intrinsèque aux aliénations de biens mobiliers religieux à partir d'observations sur deux polémiques contemporaines. D'une part, la saga judiciaire pour la restitution d'objets du «trésor» de la paroisse de l'Ange-Gardien vendus dans les années ig6o (Cour supérieure de Québec, 1980). L'affaire expose publiquement l'existence de trafics illicites d'objets d'église entre certaines autorités du culte et certains acteurs, privés et publics, du marché de l'art. D'autre part, l'analyse médiatique de la tentative de vente d'un tableau par la paroisse Notre-Dame de Québec en 2018 nous permettra d'évoquer le double standard d'exigences auquel sont soumis les musées versus les églises en contexte d'aliénation de biens patrimoniaux, ainsi que les quiproquos et mécontentements quant aux droits de l'Église de spéculer sur un patrimoine considéré collectif. La conclusion rendra compte des inconstances et zones d'ombres accompagnant le mouvement de protection des marqueurs de sacralité, alors que s'opère leur déplacement de la sphère cultuelle vers la sphère culturelle.

\section{Protection et administration du patrimoine religieux}

\section{La place de l'Église dans la société québécoise}

La structure sociale du Québec a été profondément charpentée par l'Église catholique, instance morale et spirituelle, mécène de premier ordre, et longtemps administratrice de postes publics clés. La province la plus catholique du Canada célèbre la toute-puissance divine en multipliant les églises depuis le XVII siècle et en les décorant dans le respect des principes du Concile de

\footnotetext{
I. « Cette laïcisation n'est pas incompatible avec l'existence de lois qui font référence à la religion et sont également susceptibles d'influer sur la protection et la mise en valeur du patrimoine religieux. » (Assemblée nationale du Québec, 2006, p.II).
} 
Trente. Mécènes et collectionneuses majeures, l'Église et les communautés religieuses sont d'ailleurs reconnues et continuent d'être remerciées par l'État et les musées pour leur rôle signifiant dans le développement du patrimoine artistique national sur quatre siècles (Porter, 1999, p. Io). Le clergé catholique francophone est également un éminent acteur social qui, après le Traité de Paris de 1763 cédant la Nouvelle-France à la Grande-Bretagne et le départ de l'élite coloniale vers la France, prend en charge les domaines de l'éducation et de la santé (Bergeron \& Loget, 20I8). L'Église québécoise est forte et prospère jusqu'au milieu du XXe siècle : elle anime des émissions de radio et de télévision, multiplie les implications sociales, construit de nouveaux lieux de culte et de pèlerinage. À partir des années i960, elle applique les réformes du Concile Vatican II, notamment la politique d'aggiornamento qui consiste à s'émanciper des décors classiques dont on rejette l'opulence, et à les renouveler par des commandes auprès d'artistes contemporains à la touche «simple, clair[e], authentique et adapté[e] à la technologie moderne » (Laroche, I999, p. 35). Ces mutations, bien qu'impulsées par des acteurs catholiques décidés à lutter contre un certain conservatisme de l'Église (Laperrière, I984, p. 150), n'empêcheront pas la vague de contestation contre le système conservateur et cléricaliste québécois, désignée par les sociologues comme la Révolution tranquille (Dickson, 2006).

Entre 1950 et 1970, le Québec traverse un mouvement de transition sociale impulsé par les initiatives d'intellectuels et de politiciens francophones pour moderniser, séculariser et affirmer l'identité canadienne-française. Ce mouvement social entraine une redistribution des pouvoirs économiques et politiques traditionnellement sous la tutelle du gouvernement fédéral et du clergé catholique vers l'État québécois. Cependant, plutôt que de mettre l'Église au banc, l'État développe une philosophie de laïcisation dans le respect des religions, la «laïcité-collaboration ». Ce modèle est basé sur une alliance durable entre l'État et l'Église pour mettre en valeur les racines catholiques du territoire.

À l'instar d'autres pays comme l'Espagne, l'entrée dans la modernité a pour corollaire une vague de laïcisation bousculant le paysage religieux : les taux de pratiquants, de prêtres et de religieux chutent drastiquement (Meunier, Laniel \& Demers, 20I0). La situation a un impact indéniable sur les revenus de l'Église, dont les budgets de fonctionnement sont fortement dépendants aux dons. Au Québec, les conseils de fabrique ${ }^{\mathrm{T}}$ en viennent à manquer de revenus pour veiller à l'exercice de la religion et au bon maintien de leur patrimoine (Assemblée nationale du Québec, 2005, p. 17). Les biens de l'Église, à la fois marqueurs de sacré, présages de prospérité par la vente et témoins historiques à protéger, se retrouvent au cœur de manœuvres auxquelles participent l'Église, l'État et les musées. La première faisant face aux difficultés de la prise en charge de son patri-

\footnotetext{
I. Le Conseil de fabrique, composé du curé de la paroisse et de six marguilliers laïcs élus par les paroissiens, est « propriétaire et gestionnaire des biens matériels, meubles et immeubles, servant aux activités paroissiales » (Monseigneur Couture, 1999).
} 
moine, le second y reconnaissant une utilité dans le processus de construction identitaire national (Berthold, 20I8, p. 4), alors que les troisièmes s'affirment en tant qu'experts et dépositaires des collections historiques du clergé.

\section{La protection du patrimoine religieux par l’Église}

Dans un contexte de transformations majeures, l'Église catholique du Québec reconnait et tente de parer à la dispersion de ses patrimoines. Si le phénomène de la mutation des églises est visible ${ }^{\mathrm{r}}$, la situation des biens mobiliers est beaucoup plus menacée. "Le caractère mobile de ces objets les expose aux dons, à des ventes ou encore aux vols » (Turgeon, 2005, p. 2I). La période suivant les réformes du Concile Vatican II est considérée comme une phase de désherbage sauvage menée par des curés et des conseils de fabrique dépeints comme manquant cruellement d'expertise pour évaluer les pièces qu'ils aliènent (Pelletier, I993). En I994, la Commission pontificale pour les biens de l'Église enjoint les membres du clergé à agir diligemment et à conserver les « biens culturels de l'Église », présentés comme d'importants témoins de l'histoire sociale et religieuse et de puissants intercesseurs de la foi (Leconte, 20I4, p. 42). Conséquemment, les instances religieuses vont se doter de leurs propres musées et collaborer avec des spécialistes laïcs pour préserver et faire connaitre leur patrimoine. Au Québec, il n'aura pas fallu attendre I994 pour que certaines autorités catholiques mettent en place des stratégies de protection basées sur des mécanismes de consultation et d'injonction, qui s'ouvriront de plus en plus à la collaboration avec la puissance publique.

C'est respectivement en 1937 et I970 que les diocèses de Québec et de Montréal créent leurs comités d'art sacré diocésain. L'analyse de leurs mandats, réalisée par Richard Gauthier en 2005, souligne que si Montréal concentre son action sur la sauvegarde des églises, le comité de Québec s'intéresse également au patrimoine matériel. Si à l'origine, l'objectif était de vérifier le caractère approprié des œuvres des églises, la mission de sauvegarde du patrimoine est officiellement formulée en 1992 dans les mandats du comité, qui

doit veiller à la conservation des trésors artistiques, meubles et immeubles de l'Église de Québec [et] sensibiliser les fabriques à la valeur du patrimoine religieux. (Gauthier, 2005, p. 16)

99

I. Le Conseil du patrimoine religieux du Québec (CPRQ) recense 456 églises fermées, vendues, réutilisées, transformées ou démolies depuis 1980. (CPRQ, 2016, p. 9). 
Et, l'auteur mentionne qu'en parallèle à l'action des comités, les autorités catholiques formulent des rappels à l'ordre. En avril I970, Monseigneur Roy publiait un règlement ecclésiastique de dix articles sur les devoirs et obligations des instances du diocèse de Québec concernant la conservation des œuvres d'art sacré : inventorier les œuvres classées ou jugées dignes d'intérêt, transmettre les inventaires au comité qui jugera de leur importance avec le ministère de la Culture, protéger les œuvres ou, en l'absence de conditions sécuritaires, soit les déposer au diocèse, soit les déposer ou les vendre au Musée du Québec (actuel Musée national des beaux-arts du Québec).

Toutefois, dans le contexte de restrictions budgétaires et d'augmentations des charges d'entretien des lieux de culte, les actions diocésaines semblent insuffisantes pour arrêter le trafic de l'art religieux. Dans ces circonstances, l'Église demande le soutien de l'État pour l'entretien de son patrimoine, afin qu'elle puisse se recentrer sur les activités du culte. Le rapport Risquer l'avenir: Bilan d'enquête et prospectives commandé en 1992 par l'Assemblée des évêques du Québec insiste sur la primauté de l'œuvre liturgique alors que

d'énormes sommes, initialement prévues pour la pastorale, sont détournées vers l'entretien des biens immeubles et meubles de valeur patrimoniale dans les paroisses, faute de subventions suffisantes octroyées par l'État. (Gauthier, 2005, p. 5)

À mesure de l'adaptation du discours argumentatif des autorités religieuses aux critères des subventions publiques, des représentants et experts mandatés par l'État intègrent les comités décisionnels. La coopération entre les instances religieuses et séculières s'officialise en 1995 , date de la création de la Fondation du patrimoine religieux du Québec (actuel Conseil du patrimoine religieux du Québec) et des premières mesures d'aide de l'État québécois en faveur de la restauration du patrimoine religieux (Québec, 2018, p. 61).

\section{L'appui de l'État à la sauvegarde du patrimoine religieux}

Si le patrimoine religieux catholique est considéré comme l'un des piliers de l'identité collective nationale québécoise, l'intervention de l'État dans son administration ne résulte pas d'un mouvement de privatisation ou d'appropriation des biens et lieux de culte comme cela a été le cas en France avec la Révolution, en Grande-Bretagne avec le protestantisme ou en Espagne avec les lois de désamortissement (Caparros, 2005, p. 3). L'esprit de « laïcité-collaboration » québécois passe par la reconnaissance de l'intérêt public de la conservation de certaines pièces du patrimoine religieux, historiquement, artistiquement ou ethnographiquement. On accole à certains objets de culte une fonction patrimoniale en les considérant comme « des objets de culture, des objets qui disent et qui font l'histoire de tout un peuple. » (Turgeon, 2005, 
p.I7-I8). De plus en plus conscient de l'urgence d'agir, le gouvernement confie à la Commission de la culture de l'Assemblée nationale le mandat de réfléchir à la question du patrimoine religieux. Le rapport Croire au patrimoine religieux $d u$ Québec déposé en 2006 justifie l'intervention publique dans la protection, transmission et gestion du patrimoine religieux du fait de son caractère culturel (Lucier, 2009, p. I39).

Pourtant, au Québec, l'Église catholique reste propriétaire de ses biens et le droit positif reconnait la validité des lois religieuses, notamment au regard de la protection du patrimoine des lieux de culte. Les articles I8 et 23 de la Loi sur les fabriques stipulent que toute fabrique peut « acquérir, posséder, louer, détenir, administrer et aliéner des biens, par tous modes légaux et à tout titre quelconque » et qu'elle dispose des «droits d'un propriétaire absolu » sur les dons. Mais la reconnaissance de l'intérêt public de certaines pièces du patrimoine religieux légitime l'ingérence de l'État. Le ministère de la Culture débloque des outils pour mettre en valeur ce patrimoine (subventions, inventaires, classements au Registre du patrimoine culturel du Québec ${ }^{1}$ ), en contrepartie desquels il impose un appareil de régulation tempérant les droits de propriétaire des fabriques. En vertu de la Loi sur le patrimoine culturel, le classement, qui peut être proposé par des propriétaires, des groupes citoyens ou par avis du ministère, et qui doit être entériné par la majorité des I2 membres du Conseil du patrimoine culturel du Québec, entraine l'imposition de mesures de contrôle sur le bien. L'autorisation du ministère sera nécessaire pour tout transport hors du Québec; toute altération, restauration, destruction; toute vente, cession, don visant un bien classé ; et le ministère pourra se prévaloir d'un droit de préemption².

Toutefois, les propriétaires de biens religieux sont admissibles à des subventions, comme le « Programme visant la protection, la transmission et la mise en valeur du patrimoine culturel à caractère religieux ». Entre 1995 et 2015, plus de 290 millions de dollars canadiens ont été investis dans ce fonds géré par le Conseil du patrimoine religieux du Québec (Brodeur, 2015, p. 36), un organisme intersectoriel composé de représentants religieux, d'experts et d'autorités publiques et locales. En 2018, le Plan d'action gouvernemental en culture 2018-2023 réitère l'implication gouvernementale en promettant d'insuffler 25 millions de dollars canadiens pour préserver le patrimoine culturel à caractère religieux, notamment en élaborant une stratégie de protection des biens mobiliers (Québec, 2018, p. I2).

\footnotetext{
I. En mai 2019, le Répertoire du patrimoine culturel, qui collige les données du registre, recense I375 immeubles et 26052 meubles classés, dont respectivement $24 \%$ et $6,4 \%$ de facture religieuse. Si le moteur de recherche ne permet pas de distinguer selon les religieux, le survol des résultats rend compte d'une nette prédominance du patrimoine catholique.

2. «Le ministre peut acquérir tout bien patrimonial classé [...] de préférence à tout autre acheteur au prix offert par ce dernier. » (RLRQ, c P-9.002, art. 56)
} 


\section{Les actions des musées pour le patrimoine religieux}

Les troisièmes piliers de la protection du patrimoine d'Église sont les muséologues. Au-delà d'être reconnus comme des dépositaires fiables et investis par l'État de missions de valorisation du patrimoine religieux ${ }^{1}$, le mouvement de laïcisation de la deuxième moitié du $\mathrm{XX}^{\mathrm{e}}$ siècle promulgue les musées nouveaux gardiens de l'art et du patrimoine catholique (Lacasse et Porter, 2004). Cependant, les attitudes et les pratiques ont largement évolué au cours des cinquante dernières années.

En 1978, Gérard Lavallée, alors président de la Société des musées du Québec, consacrait l'éditorial du deuxième numéro de la revue Musées à l'action bienfaitrice du milieu muséal face à la «dilapidation » du patrimoine ecclésiastique. Il dénonçait le silence des autorités civiles et religieuses dans les années i960, alors que les collectionneurs et les musées

alertés par cette situation dramatique firent l'impossible pour récupérer quelques-uns de ces objets. Et c'est grâce à eux, aujourd'hui, si un peuple tout entier peut se reconnaitre dans la qualité [du] passé (...). (Lavallée, 1978).

Le collectionnement est justifié par des considérations artistiques ou historiques et une visée nationaliste, notamment chez Gérard Morisset, qui réalise entre 1937 et 1969 un recensement exhaustif du patrimoine artistique ancien du Québec. Son initiative vise particulièrement le patrimoine des églises et communautés religieuses (Musée du Québec, I98I) et ce, dans le but de remédier au «pillage de la Province » par les Ontariens et les Américains (Derome, 1984, p. 23) au profit du Musée des Beaux-arts du Québec qu'il dirigera, ainsi que de l'ensemble du réseau muséal québécois.

D'uniques sauveurs, le discours des muséologues s'adoucit et les conservateurs du XXI ${ }^{e}$ siècle présentent les musées comme un « outil supplémentaire » (Des Rochers, 2005, p. I67) pour étudier, diffuser et transmettre les sens du patrimoine religieux et mieux comprendre l'histoire québécoise. Les musées y investissent une part importante de leur budget d'acquisition et d'exposition dans un certain climat de compétition (Des Rochers, 2005, p. I65). Au-delà d'être acquéreurs, ils collaborent avec les communautés religieuses via des ententes de prêts, de dons et de dépôts (Porter, 2005, p. 157). De plus, en 2012, les États généraux des musées du Québec réitéraient formellement une série d'actions à entreprendre conjointement : inventorier, tenir à jour et numériser les col-

\footnotetext{
I. On pense au Règlement diocésain de r970 susmentionné ou à la requête du Premier ministre pour que le Musée des beaux-arts de Québec organise l'exposition Le Grand Héritage lors de la visite du Pape Jean-Paul II en 1984 .
} 
lections ecclésiastiques; permettre aux musées de développer leurs collections et de poser des actions en faveur du patrimoine religieux; se doter d'un plan d'action concerté pour la sauvegarde et la conservation du patrimoine mobilier religieux en impliquant le Conseil du patrimoine religieux, la Société des musées du Québec, les musées et le ministère de la Culture.

Basée sur des actions ciblées et des mécanismes de concertation, la politique québécoise de protection du patrimoine religieux est originale par rapport aux approches nationalistes, injonctives et impératives d'autres pays. Cependant, deux cas polémiques d'aliénations démontrent les failles du système en révélant certaines incohérences des politiques de gestion des biens de l'Église.

\section{Polémiques autour d'aliénations de biens de l'Église}

\section{L'affaire de la paroisse de l'Ange-Gardien et l'incessibilité des choses sacrées}

En 1976 , la fabrique de la paroisse de l'Ange-Gardien intentait une action devant la Cour supérieure du Québec (CSQ) en restitution d'objets sur le fondement de l'illégalité de leur vente par un curé en $1963^{\circ}$. La fabrique poursuivait : le curé de l'époque, le premier acquéreur ainsi que les trois collectionneurs et deux musées canadiens possédant les objets au moment du procès, soit le Musée national des Beaux-arts du Québec et le Musée des Beaux-arts du Canada (MNBAC et MBAC). Les arguments de la requérante étaient de trois ordres : le curé n'avait pas la capacité de vendre les objets de la paroisse, et faute d'avoir été désacralisés, les objets en question étaient non-commercialisables. Ce procès, unique en la matière au Québec, clarifie le régime de propriété des choses sacrées, explicite les procédures et compétences nécessaires pour aliéner ces objets, et met en lumière les manœuvres douteuses du marché de l'art religieux.

L'une des difficultés du régime de propriété s'appliquant au patrimoine religieux est de qualifier la sacralité d'une chose. En effet, différentes catégories de biens matériels cohabitent dans les lieux sacrés catholiques. Pensons aux restes humains (reliques), aux objets nécessaires aux rituels (calices, bénitiers), utilitaires (chaises, pupitres) et décoratifs (tableaux, sculptures). Pour qualifier le caractère des biens ciblés par le procès, le juge saisi de l'affaire renvoie aux lois religieuses visant la sacralité (CSQ, 1980, \$249). Or, le titre « Les contrats et en particulier l'aliénation » du Code de droit canonique se rapporte au « patrimoine stable », sans en distinguer les composantes sacrées, et le canon I2 Iо considère que la présence d'objets dans les lieux de culte est conditionnelle à leur usage religieux. Le juge présume donc tout objet d'Église sacré par destination ${ }^{2}$, en précisant que la qualité artistique des choses n'annule pas leur caractère sacré (CSQ, I980, §286).

I. Au titre des biens visés : calices, encensoirs, navettes, bénitier, burettes, ampoules pour Saintes Huiles, aiguière, crucifix, chandeliers, statues, porte de tabernacle, portes de fonts baptismaux, tambour sculpté, vêtements sacerdotaux (CSQ, 1980, §2I).

2. En 1993, Benoît Pelletier précise qu’une chose sacrée peut être hors commerce en raison de sa 
Qu'ils soient sculptures ou calices, les biens au cœur du litige sont considérés par le juge comme sacrés et, à ce titre, dotés d'une protection spéciale. Les gestionnaires ne peuvent en disposer comme ils le feraient du patrimoine $\mathrm{du}$ « commun des mortels» (Derome, I984, p. 2I). Ils sont hors commerce, incessibles et imprescriptibles au sens de l'article $2217 \mathrm{du}$ Code civil du Bas-Canada ${ }^{\text {I }}$ Or, la présomption de sacralité fait peser la charge de la preuve sur la défense, qui doit démontrer la désacralisation et la légalité de la transaction d'achat, et ce, dans le respect des procédures énoncées par le droit religieux compétent. En l'espèce, le canon I293, qui exige de démontrer une juste cause d'aliénation (comme une urgente nécessité, une évidente utilité, la piété, la charité ou toute autre grave raison pastorale); d'avoir une expertise écrite du bien ; et de respecter toutes autres précautions, notamment l'obtention des consentements écrits nécessaires. Il faut donc être en mesure de démontrer la décision collective des membres du Conseil de fabrique autorisant la vente ${ }^{2}$, et l'autorisation émanant d'une autorité supérieure lorsque l'aliénation porte sur un bien présentant un intérêt historique ou artistique ou acquis il y a plus de 50 ans (Loi sur les fabriques, art. 26, §i) 3. En l'espèce, l'évêque aurait dû émettre une autorisation écrite. Faute de prouver l'existence d'actes autorisant le curé à aliéner les objets, le juge conclut que le caractère sacré des biens n'a pas été révoqué : ils étaient donc hors commerce (Caparros, 2005, p. 5). Par conséquent, la fabrique est reconnue «seule, véritable et unique propriétaire ", les actes de vente sont déclarés nuls et les défendeurs doivent restituer les biens en leur possession.

Ce procès a la particularité de rendre compte, entre les lignes, mais fort publiquement, de l'existence de trafics illicites réalisés en toute impunité. En effet, en cours de procédure, la défense tente de faire valoir l'existence d'un usage coutumier qui octroierait aux curés les pleins droits de disposer des objets des paroisses (CSQ, I980, \$25). L'argument est réfuté par le juge, faute de démontrer la valeur coutumière - c'est-à-dire les caractéristiques ancienne, fréquente, générale, publique et uniforme de la pratique - notamment parce que les preuves rapportées ne sont pas plus anciennes que les années I960. Pourtant, cette ligne argumentative expose sans doute l'imprécision des cadres légaux, en tout cas la banalité de pratiques illégales. La bonne foi des acquéreurs laisse à désirer. Bien qu'ils aient tous eu connaissance de la provenance des objets, aucun ne s'est inquiété du régime légal applicable. De tels biens étant à

nature (la sacralité des corps morts) ou de sa destination, c'est-à-dire par l'usage religieux de la chose. I. Cet article n'a pas été repris dans le Code civil du Québec de r99r. Mais l'article 696 du Code de procédure civile énonce l'insaisissabilité des « vases sacrés et autres objets servant au culte religieux », et l'article 2876 du Code civil dispose que «(c)e qui est hors commerce, incessible ou non susceptible d'appropriation, par nature ou par affectation, est imprescriptible. »

2. La décision d’aliéner ne peut, en aucun cas, être du seul ressort du curé (CSQ, 1980, \$273).

3. Dépendamment de la valeur des biens, l'autorité en question va de l'évêque (jusqu'à 550000 dollars canadiens) au Saint-Siège du Vatican (plus de 5,5 millions de dollars canadiens) (Conférence des évêques catholiques du Canada, 2018). 
présumer sacrés, les acquéreurs auraient dû s'assurer du respect des procédures de désacralisation et d'aliénation (CSQ, 1980, \$285). Notons également les manœuvres des musées et des administrateurs publics présents dans la chaîne de transactions, et manquant à ce titre de ce que l'on qualifie aujourd'hui de diligence raisonnable ${ }^{\mathrm{r}}$. En date de 1967 , une lettre de Pierre Théberge, conservateur au MBAC à son homologue Jean Trudel du MBAQ fait part de doutes sur la légalité de la situation. Ce qui n'empêchera ni Québec ni Ottawa d'acquérir des objets de la paroisse, au prétexte de la sauvegarde de ce patrimoine.

[L]a statue aurait quitté l'église en 1963 et je crois bien quil s'avérait que son «départ "n'ait pas été légal, la Galerie nationale, outre qu'elle se trouverait dans une situation quelque peu embarrassante, songerait sans doute à en faire la restitution à son propriétaire légitime. (CSQ, 1980, \$50)

\section{9}

La forte médiatisation de ce procès, confirmé par la Cour d'appel du Québec en 1984 , aurait, selon certains auteurs, entrainé un mouvement de responsabilisation et de prudence chez les acteurs du marché des objets d'églises (Gauthier, 2005; Pelletier, 1993). Pour Richard Gauthier, si l'affaire a souligné certaines rivalités, elle s'est soldée par une consolidation des politiques de concertation entre les pouvoirs laïcs et religieux visant des résolutions plus confidentielles des écarts en contexte d'aliénation. Pourtant, un bond dans le temps nous montre la survivance de malentendus entre les acteurs de la protection du patrimoine religieux.

\section{L'affaire de la paroisse Notre-Dame de Québec et la vente du patri- moine religieux}

Le 4 avril 20I8, quelques jours après la parution du catalogue de vente de Christie's, les médias canadiens annonçaient la décision du MBAC d'aliéner une ouvre de Marc Chagall, et ce, pour acquérir « un trésor national». Dans une lettre aux médias datant du I7 avril, Marc Mayer, alors directeur général du Musée, justifie cette cession comme la seule option permettant de lever les fonds nécessaires à l'acquisition d'un tableau risquant de quitter le pays. La toile en question est Saint-Jérôme (I779-1780) de Jacques-Louis David dont la fabrique de la paroisse Notre-Dame de Québec cherche à se départir. Fortement médiatisé, ce cas rend compte du double standard de l'opinion publique face aux aliénations, et certaines dissensions quant à la propriété (privée ou publique) du patrimoine religieux.

\footnotetext{
I. Le «(d)egré de prudence (...) auquel on peut à bon droit s'attendre de la part d'une personne raisonnable et prudente (...) dans une situation donnée. » (Société des musées du Québec, en ligne)
} 
L'analyse des articles de presse canadiens ${ }^{\mathrm{r}}$ révèle les différents degrés d'attente auxquels sont soumises les instances muséales et ecclésiastiques. Du côté du MBAC, l'annonce de la vente d'une toile de Chagall évaluée entre 6 et ro millions de dollars américains a soulevé une série de critiques. Le musée considère que l'aliénation du tableau décrit comme manquant de qualité muséale perfectionnera la collection en permettant d'acquérir la toile religieuse mise en vente par la fabrique de Québec. Mais les opposants à la vente sont nombreux - mentionnons la marchande Miriam Shiell, la critique Manon Gauthier, l'expert Alexander Herman, le collectionneur Bruce Bailey - et les journalistes particulièrement mordants. Ils reprochent le manque de transparence ${ }^{2}$, l'attitude défiante du directeur ainsi que le climat de rivalité alors que le MBAC fait face à l'alliance de deux musées québécois. Bien que l'aliénation soit en théorie une pratique de gouvernance admise dans les musées canadiens, on constate que manifestement, la marchandisation de l'ouvre d'un artiste de renommée internationale ne passe pas. Face à la controverse, le musée annule la vente alors que l'opinion publique réclame un encadrement plus strict de la gestion du patrimoine national. De l'autre bord, la presse présente la fabrique Notre-Dame de Québec comme la propriétaire d'une toile de David suite à son don dans les années i930. Elle l'aurait fait expertiser, proposée à plusieurs musées canadiens pour 6,3 millions de dollars canadiens (Clément, 20r8) et faute d'offre satisfaisante, aurait entrepris des démarches pour la vendre à des institutions outre-Atlantique (Bouchard, 20I8). La presse anglophone ne compte qu'un éditorial critiquant l'action spéculative de la fabrique (Globe and Mail, 20I8). Du côté francophone, ce comportement est remis en question après la parution d'articles évoquant le devoir de conservation de l'Église que signent des collectionneurs et chercheurs (Nadeau, 2018 ; Joyal \& Lambert, 20I8; Dubé, 20I8) : l'Église n'aurait moralement pas le droit de spéculer sur les biens patrimoniaux dont elle ne serait que la dépositaire et gardienne. Cela contraint le porte-parole, Monseigneur Bélanger, à clarifier les droits de propriétaire de la fabrique, pour ensuite annoncer, le 3 mai 20I8, l'intention de la paroisse de « laisser la poussière retomber avant de rediscuter avec les musées » (Bussières, 20I8).

La couverture de l'affaire montre une confusion entre les notions de «patrimoine collectif » et de "propriété publique ». À la suite de Philippe Dubé et Laurier Lacroix, plusieurs journalistes et la ministre de la Culture se demandent s'il est légitime que les contribuables québécois payent pour qu'un tableau continue d'être exposé, alors qu'il aurait été donné à la paroisse à cette fin. L'argumentaire reprend la thèse qu'avancent Luc Noppen et Lucie K. Morisset dans les années 2000, en considérant le patrimoine religieux comme une propriété collective sur laquelle l'Église ne devrait pas pouvoir spéculer. Pour

\footnotetext{
I. Soit 54 articles anglophones et IO4 articles francophones publiés entre le $\mathrm{I}^{\mathrm{er}}$ avril et le 6 juin $20 \mathrm{O} 8$ et colligés par la base de données Eureka.

2. Sont reprochées la culture du secret, l'absence de consultation publique, l'annonce de la vente par Christie's.
} 
Ernest Caparros, le terme « patrimoine collectif» porte à confusion en sous-entendant que la gestion des biens de l'Église «puisse être décidée, en partie, à l'extérieur de ses rangs. » (Assemblée nationale du Québec, 20o6, p. 8). Le régime de propriété ne doit pas être confondu avec l'intérêt public de certains objets. Rappelons que, sur le plan juridique, le don transfère l'intégralité des droits de propriété au donataire, ici la paroisse, à moins de spécifications contraires (Caparros, 2005, p. 6). Or, en avril 2018, la toile n'était pas classée au Registre du patrimoine culturel du Québec. La seule restriction dépendait d'une convention de dépôt signée en 1995 au moment du transfert de la gestion du tableau au Musée de la civilisation de Québec, lui ménageant un délai de six mois pour faire une contre-offre en cas de vente.

Pourtant, plusieurs arguments sont appelés à justifier de l'importance collective du tableau : il aurait un intérêt historique et artistique démontré par le fort sentiment d'appartenance soulevé par l'affaire; il aurait été donné à la paroisse par des Québécoises, déposé, conservé et exposé par des musées québécois, et entretenu, à titre de patrimoine d'église, par des subventions publiques et des dons caritatifs québécois. C'est sur ces fondements - même si certains sont questionnables ${ }^{\mathrm{I}}$ - qu'intervient le ministère de la Culture. Le 23 avril, la ministre émet un avis de classement qui lui permet de contrôler l'exportation du bien et, advenant d'une vente hors du Québec, de le retenir temporairement sur le territoire ${ }^{2}$. L'Église, par l'entremise de Monseigneur Bélanger, insiste pour que son droit de propriétaire soit respecté, tout en observant certaines défaillances du soutien gouvernemental, nommément la perte d'une subvention canadienne. Mais l'image de collaboration entre l'État et l'Église demeure, puisqu'il salue l'initiative de classement de la toile par le ministère québécois (Bussières, 2018).

\section{Conclusion}

Au Québec, la protection du patrimoine catholique justifie des mesures allant du radical - au point de transgresser les règles d'une Église dont on annonçait la disparition - à des actions concertées, mais avouées à demi-mot, alors que spécialistes et autorités religieuses sont sensibilisés à la protection du patrimoine et informés de la valeur des biens. Le passage de la prise en charge du patrimoine religieux des églises aux musées laisse deviner des zones d'ombres, alors que le déplacement des objets répond d'une pléiade de stratégies dont il est regrettable de constater qu'elles manquent parfois de rigueur, de clarté et de transparence. Pour autant, si la muséalisation de ces biens entraine leur conservation ex situ, elle semble, à ce jour, être la stratégie la plus adaptée pour protéger les œuvres d'intérêt public puisque les musées ont plus d'influence et de compte à rendre que l'Église (Bergeron \& Loget, 20I8). Toutefois, la méthode scientifique et l'ambition de possession des musées produisent d'une part,

I. Voir Routhier (2006) sur le sophisme de l'argumentaire financier.
2. La toile sera classée le II avril zorg à titre d'objet patrimonial. 
un discours disciplinaire sur le patrimoine sacré qui a tendance à oblitérer l'interprétation théologique d'objets cultuels, vivants et porteurs de charges immatérielles, et d'autre part, une incompatibilité avec l'usage liturgique et la présentation des objets sacrés dans leurs environnements d'origine. Si les journaux s'intéressent aux cas polémiques, tels une toile de plusieurs millions ou les crucifix trônant aux murs de tribunaux, de conseils municipaux et d'Assemblée nationale, espérons que les discussions à venir entre le ministère, les autorités ecclésiastiques et les experts, émergent sur des stratégies de recollement et de mise à jour des inventaires de biens mobiliers à caractère religieux ; ainsi que des mécanismes de responsabilité collégiale et de transparence quant à la gestion de ce patrimoine, in situ et ex situ, notamment lorsqu'il est question d'aliénation.

\section{Références}

Arpin, R. (Dir.). (200o). Notre patrimoine, un présent du passé. Québec, Canada : Groupe-conseil sur la politique du patrimoine culturel du Québec.

Assemblée nationale du Québec. (2006). Croire au Patrimoine religieux du Québec. Québec, Canada: Assemblée nationale.

Assemblée nationale du Québec. (2005). Patrimoine religieux du Québec. Document de consultation. Québec, Canada: Assemblée nationale.

Bergeron, Y. \& Loget, V. (2018). Du désenchantement au réenchantement : le cas du Saint-Jérôme de David. Dans F. Mairesse (dir.). Museology and the Sacred Materials for a discussion, (pp. 33-38). Paris, France : ICOFOM.

Berthold, É. (Dir.). (2018). Le patrimoine des communautés religieuses : empreintes et approches. Québec, Canada : Presses de l'Université Laval.

Bouchard, G. (I6 avril 20ı8). Un Chagall pour un David...ou pas. Le Soleil, 37.

Brodeur, M. (Dir.). (2015). 20 ans d'action. Un parcours rassembleur. Québec, Canada : CPRQ.

Bussières, I. (3 mai 2018). La paroisse estime être bel et bien propriétaire du Saint Jérôme, Le Soleil, 22.

Caparros, E. (2005). La propriété des églises catholiques au Québec. Présentation au colloque Quel avenir pour quelle église?, Montréal 19-22 octobre 2005. Page consultée le 2 février 2019, au http://bibliotecanonica.net/ docsaj/btcajl.pdf.

Clément, É. (I8 avril 20I8). Québec évoque un classement patrimonial. La Presse. Page consultée le 2 février 2019 au http://plus.lapresse.ca. 
Conférence des évêques catholiques du Canada. (mars 20I8). Décret 38 : Somme maximale pour l'aliénation des biens ecclésiastiques. CCCB. Page consultée le 2 février 2019, au http://www.cccb.ca/site/images/stories/pdf/ Biens_ecclesiastiques_-_20I8.pdf.

Commission pontificale pour les biens culturels de l'Église (Io avril 1994). Les biens culturels des institutions religieuses. Saint-Siège. Page consultée le 2 février 2019, au http://www.vatican.va/roman_curia/pontifical_commissions/pcchc/documents/rc_com_pcchc_index-documents_fr.html.

Cour supérieure de Québec (1980). L’Ange-Gardien (Paroisse) c. Québec (Procureur général). CSQ no 200-05-001510-76. La Référence. Page consultée le 2 février 2019, au https://www-lareference-editionsyvonblais-com.

Couture, M. (1999). L'église, une institution comme les autres ? Oui...et non !. Présentation à la conférence Déjeuner-conférence de l'Institution d'administration publique de Québec. Page consultée le 9 mai 2019, au http://www. iapq.qc.ca/wp-content/uploads/20I2/og/conf_MCouture4_O3_I999.pdf.

CPRQ (2018). Rapport annuel 2017-2018. Québec, Canada : CPRQ.

CPRQ (2016). Mémoire déposé dans le cadre des consultations publiques portant sur le renouvellement de la politique culturelle du Québec. Québec, Canada : CPRQ.

Derome, R. (1984). L'art sacré : Une étude de gestes. Continuité, 25, I8-24.

Des Rochers, J. (2005). Mise en valeur du patrimoine religieux au Musée des beaux-arts de Montréal. Dans L. Turgeon, Le patrimoine religieux du Québec : entre le cultuel et le culturel, (pp. I6I-I69). Québec, Canada : Presses de l'Université Laval.

Dickson, O. (2006). La Révolution tranquille : période de rupture ou de continuité. Montréal : UQAM, thèse de maîtrise en sciences politiques.

Dubé, P. (27 avril 20I8). L'Église doit rendre le « Saint-Jérôme » à la population. Le Devoir, A9.

Église catholique. Code de droit canonique. Saint-Siége. Page consultée le 2 février 2019, au http://www.vatican.va/archive/FRAoo37/_INDEX.HTM\#fonte.

Gauthier, R. (2005). Le Devenir de l'art d'Église dans les paroisses catholiques du Québec. Québec, Canada : Presses de l'Université Laval.

Globe and Mail (25 avril 2018). When you mix art and politics, strange things happen, The Globe and Mail. Page consultée le 2 février 2019, au https:// www.theglobeandmail.com/opinion/editorials/article-globe-editorialwhen-you-mix-art-and-politics-strange-things-happen/.

Joyal, S., \& Lambert, P. (I9 avril 2018). La vente du «Saint Jérôme » un imbroglio toxique. Le Devoir, A7. 
Lacasse, Y., \& Porter, J. (Dir.). (2004). Une histoire de l'art du Québec. La collection du Musée national des beaux-arts du Québec. Québec, Canada : Musée national des beaux-arts du Québec.

Laperrière, G. (1984). L'adaptation à de nouveaux modes de vie. Dans Trudel, J. (dir.), Le Grand héritage : l'Eglise catholique et la société du Québec (pp. I29-170). Québec, Canada: Musée du Québec.

Laroche, G. (Dir.). (1999). Le renouveau de l'art religieux au Québec 1930-1965, Québec, Canada: Musée du Québec.

Lavallée, G. (1978). Les musées et la récupération du patrimoine. Musées, I(2), I.

Leconte, A. (20I4). Hors de l'église, point de salut? La transmission du sens des œuvres d'art religieux. Rabaska, I2, 39-56.

Lucier, P. (2009). La signification culturelle du patrimoine religieux. Dans S. Lefebvre (Dir.), Le patrimoine religieux du Québec. Éducation et transmission du sens, (pp. I39-I49). Québec : Presses de l'Université Laval.

Meunier, E.-M., Laniel, J.-F., \& Demers, J.-C (2010). Permanence et recomposition de la « religion culturelle ». Dans R. Mager \& S. Cantin (Dir.). Modernité et religion au Québec, (pp. 79-I28). Québec, Canada : Presses de l'Université Laval.

Minucciani, V. (Dir.). (2013). Religion and Museums. Immaterial and Material Heritage. Turin, Italie : Umberto Allemandi \& C.

Musée du Québec (198I). À la découverte du patrimoine avec Gérard Morisset. Québec, Canada : Ministère des Affaires culturelles et Musée du Québec.

Nadeau, J-F. (I4 avril 20I8). Le nébuleux commerce des ouvres du patrimoine religieux. Le Devoir. Page consultée le 2 février 20i9, au https:// www.ledevoir.com/culture/arts-visuels/525218/le-nebuleux-commerce-des-oeuvres-du-patrimoine-religieux.

Paine, C. (20I4). Sacred Waste. Material Religion, IO(2), 24I-243.

Pelletier, B. (1993). The Case of the Treasures of L'Ange Gardien: An Overview. International Journal of Cultural Property, 2(2), 371-382.

Porter, J. R. (1999). Un art religieux à l'enseigne de la modernité. Dans G. Laroche (dir), Le renouveau de l'art religieux au Québec 1930-1965 (pp. 7-Io). Québec, Canada: Musée du Québec.

Porter, J. R. (2005). L'avenir de notre patrimoine religieux par-delà les urgences du temps présent : le rôle et l'engagement du Musée national des beauxarts du Québec. Dans L. Turgeon (dir.), Le patrimoine religieux du Québec: entre le cultuel et le culturel. Québec, Canada : Presses de l'Université Laval 
Québec. Code civil du Québec, RLRQ c CCQ-1991. Legisquebec. Page consultée le 2 février 20I9, au http://legisquebec.gouv.qc.ca/fr/showdoc/cs/CCQ-I99I.

Québec. Loi F-1 sur les fabriques. Legisquebec. Page consultée le 2 février 2019, au http://legisquebec.gouv.qc.ca/en/ShowDoc/cs/F-r?langCont=fr.

Québec. Loi sur le patrimoine culturel, RLRQ c P-9.0oz. Legisquebec. Page consultée le 2 février 2019, au http://legisquebec.gouv.qc.ca/en/ShowTdm/ cs/P-9.002?langcont=fr.

Québec (2018). Partout la culture. Plan d'action gouvernemental en culture 20182023. Page consultée le 9 mai 2019, au https://mcc.gouv.qc.ca/fileadmin/ documents/Politique_culturelle/Plandactionculture20182023_web.pdf.

Québec. Répertoire du patrimoine culturel. Page consultée le 9 mai 2019, au http:// www.patrimoine-culturel.gouv.qc.ca.

Roque, M. I. (20II). Le musée, lieu de mémoire du patrimoine religieux. Les nouvelles de l'ICOM, 3 .

Routhier, G. (2006). Les enjeux du débat actuel sur le patrimoine religieux. Argument. Page consultée le 2 février 2019, au http://www.revueargument. ca/article/2006-03-or/347-les-enjeux-du-debat-actuel-sur-le-patrimoinereligieux.html.

Société des musées du Québec (2012). États généraux des musées du Québec : Bilan et prospective. Musées, 30, 40-59.

Société des musées du Québec. La gouvernance des institutions muséales : guide à l'usage des directions et des conseils d'administration. Page consultée le I2 mai 2019, au https://www.musees.qc.ca/bundles/professionnel/guidesel/ gouvernance/lexique/index.htm.

Trudel, J. (Dir.). (1984). Le Grand Héritage : l'Eglise catholique et la société du Québec. Québec, Canada: Musée du Québec.

Turgeon, L. (Dir.). (2005). Le patrimoine religieux du Québec : entre le cultuel et le culturel. Québec, Canada : Presses de l'Université Laval. 